\title{
The Impact of Immersive Virtual Reality on Visualisation for a Design Review in Construction
}

\author{
May Bassanino ${ }^{1}$, Kuo-Cheng Wu, Jialiang Yao, Farzad Khosrowshahi, Terrence Fernando, and \\ Jens Skjærbæk ${ }^{2}$ \\ University of Salford, Salford, United Kingdom \\ ${ }^{2}$ COWI A/S Aalborg, Denmark \\ \{m.n.bassanino@salford.ac.uk, kuo.cheng.wu@gmail.com, j.yao@ salford.ac.uk, \\ f.khosrowshahi@salford.ac.uk, t.fernando@salford.ac.uk, JEO@cowi.dk\}
}

\begin{abstract}
Communication during the design process has a substantial role because; it exchanges messages and conveys ideas to people with different skills and interests. Also, the development of high quality $3 D$ modelling, photo rendering and VR software has shifted the way we communicate architecture, from a conventional one to a digital format and so, provided us with another way for the communication of information, the visualisation of processes and the creative expression of ideas.

This paper investigates the impact that Immersive Virtual Reality technology can have on visualisation of a design review scenario in construction, which was developed during the course of the European CoSpaces project and the potential of such technologies to alter the way enterprises work.
\end{abstract}

Keywords- Immersive VR, communication, current practice, future scenario, CoSpaces

\section{Introduction}

The relationship between architectural representation and its intended final product; the building has undergone a profound transformation over the centuries. The age of computer aided-design has brought another dimension to architectural presentation. With the rapid development of computer hardware and software, we became capable of producing images with various kinds of presentation techniques [1]

For the nature of the construction industry and its projects, a typical construction project usually consists of an amalgamation of both direct and indirect stakeholders representing a diversity of skills, cultures and disciplines [2]. However, these stakeholders need to work together to deliver the final product and therefore a tremendous amount of collaboration is required to ensure that all those involved share the same understanding about the project. Since "shared thinking, shared planning and shared creation" are essential elements for any collaboration [3] communication is therefore very fundamental in the success of any project [4] [5]

This paper will discuss a futuristic design review in construction where a number of project team come together in a single technology driven meeting space to redesign a disabled bathroom in a block of flats. It will first explain the current practice and then examine the impact that immersive environment can have on communicating design among the various stakeholders involved in the project. In other words, the work investigates whether the digital model can replace the physical model or not.

\section{Communicating Design}

$75 \%$ of each working day is spent in some form of communication [6] Whether it is verbal or visual, communication is the centre activity of any project. In an architectural context, the transmission of information to communicate design through visual representation of the real world is very crucial; there is no doubt that presentation techniques can play a major role in how we perceive design [1] as different presentation techniques can easily alter our perception of architecture [7] [8]

It is the architect's concern to communicate design and convey his/her ideas to various stakeholders in the project team; it is important to select the appropriate technique to present the architectural design as the keystone in communicating design intentions is to convey much information about what it feels to experience the real building; good buildings exceed our expectations while poor designs look worse in real life [9] .

Communicating architecture in a conventional way whether by producing drawings or a physical mock up has been accepted for a very long time. The act of 
drawing has been considered as the means by which architects manifest their contribution to a design [10] .

The advancing Computer Aided Design gave us the opportunity that architecture could be communicated digitally in a variety of formats such as still or animated images, VR, multimedia and others. These digital technologies added the effect of sound, images, words, gestures and even expressions when paper exchanges messages via a single sense which is seeing [1] CAD has therefore started being used as a communication tool during the design process [11] which started creating the potential for a new way to convey design ideas which ultimately result in more cooperation and better shared understanding of design intentions.

\section{The CoSpaces Case Study of a Design Review in Construction}

The CoSpaces multi million European project's aim was to develop a generic collaborative and engineering environment which can support real-time collaboration between geographically dispersed teams working irrespective of their location [12] .

During the course of CoSpaces, a number of case studies were developed with the aim to express a realistic vision of the industry concerning the way advanced technologies could support collaboration in construction projects. These case studies provided an understanding of the current situation as well as the problems of collaborative working. In addition, the case studies investigated the use of technologies to enable a better working practice through first, providing a set of tools to assist project teams to communicate and collaborate in a more efficient way and secondly, these case studies investigated how technologies such as Virtual Reality, Augmented Reality and others can help in visualisation of the design and investigation of different alternatives and layouts.

The design review scenario in construction which is the focus of this paper is about a space that was originally designed to be a bathroom for disabled people. During construction, the bathroom space was reduced in floor area, because of the addition of a separate installation shaft for the supply of a ventilation system in the space in order to respond to new requirements for fire protection and safety. As a consequence, the bathroom has to be redesigned, but must include the same elements as previously planned: a close-coupled WC, a basin, a bath, a wall hung cupboard and a window [13] .

\subsection{Current Practice (as is)}

A meeting is required to discuss the layout for a disabled bathroom in a block of flats as illustrated in figure 1. The organisation of the meeting takes time in order to find a common available date of all the participants. Once a date is fixed and all the stakeholders confirm their availability, a physical mock up is then built at the architectural company for use during the meeting.

The mock-up has already been tested by the architect, the engineers and the main contractor in order to achieve a good level of certainty that the meeting would validate their proposition. A wheelchair user was invited to test the usability of the bathroom and that the elements are within reach and usable. While everyone is discussing the current design and trying to consider any future issues that could arise from it, the wheelchair user starts testing the mock-up. Immediately, the end-user realises that the bathroom space is insufficient for a wheelchair to manoeuvre and some elements are not accessible, and therefore more space is required. Due to the fact that the bathroom has to be fully redesigned, a modified physical mock-up should be created before any validation is possible.

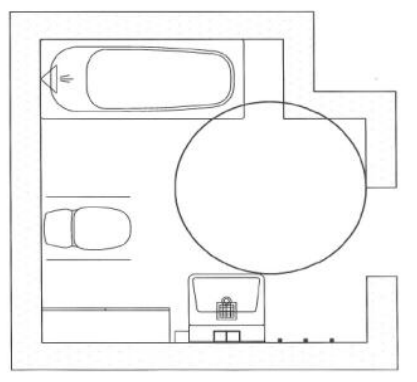

\section{Figure 1 Current layout for the disabled bathroom}

The meeting ends with some limited discussions between all the participants in order to achieve a valid design for the next meeting. The architect takes some notes on his notebook and quickly annotates some drawings. It is discussed that one possibility is to exchange the bathtub with a shower arrangement- it cannot be decided what type of shower should be used, but the end-user has some basic criteria such as an even floor level, the possibility to sit, and the need for a panic button. Finally, it was agreed that another two hour meeting will be scheduled approximately a week later with the same participants.

By now, the project cost has already increased by the cost of the physical mock-ups and the travel expenses, as well as the cost of the staff time and materials spent redesigning the bathroom.

\subsection{Future Scenario (could be)}

The futuristic scenario proposed here corresponds to the same situation as the one described above. It illustrates the use of new technologies to improve meetings through better communication and visualisation [13] [14] . Its objective is to make the meetings more effective, which means that there is a better shared understanding between the participants, that more viewpoints can be considered and problems can be 
resolved much faster. In order to achieve this, useful information has to be made available faster between all the participants, in a way that is easily understood by the people who need it. As a consequence, fewer meetings will be required due to incomplete agreements, fewer problems have to be solved and the possibility of redesigning as well as testing alternative solutions during the meeting will be available to speed up the building construction.

Similar to the previous project situation, the project manager invites the relevant stakeholders to attend a meeting at the architectural company to discuss the new layout.

On the day of the meeting, as the participants connect to their workstation, the architect starts the meeting by identifying the problem and suggesting some alternative design solutions. After the presentation, the participants study the design proposed by the architect and discuss the various alternatives sharing their views.

In the next phase of the meeting, a wheelchair user tests the accessibility of the bathroom (figure 2). Similar to the previous scenario, he finds the space is restricted and there is no accessibility for a wheelchair to manoeuvre.

Immersive Virtual Reality is used here to produce the digital mock up in an attempt to replace the physical one in this scenario, so the meeting does not end here, but it carries on where changes can be applied by the various stakeholders as they discuss their viewpoints. Once all changes are made, the wheelchair user starts testing the bathroom's model for the second time. Once an acceptable solution for a new design layout is agreed, the meeting ends with a definitive validation of the design, and the participants can then return to their everyday work without the need for further meetings to be organised and for physical mock-ups to be modified.

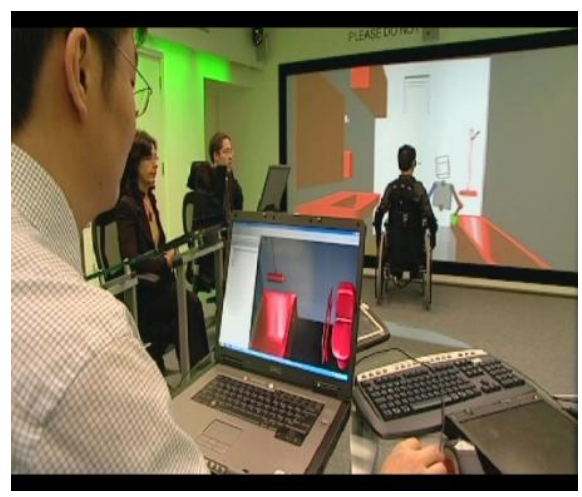

Figure 2 The wheelchair user is testing the bathroom's layout using the digital mock up during the meeting

\section{The CoSpaces framework}

The framework developed in the CoSpaces EU project consists of several main modules such as
Collaboration Broker (CB), Dynamic Session Manager (DSM), Knowledge Support Management (KSM) (KSM), Resource Manager and Application Controller (AC).

During the meeting, all participants used their laptops with the CoSpaces system installed on each machine. They used the Living Lab Infrastructure, referred to Active Distributed Development Space (ADDS). The system ran on Windows XP operating system and the laptops were equipped with current standard CPU/RAM/graphic card. A plasma display if available in the meeting room could be used to project the design of the selected participant's workspace to facilitate interactive brain storming sessions among the meeting's participants.

The physical system set up (figure 3) consisted of: model data server, several desktop clients to support the multi stakeholders in the project team (such as Architect, Structural Engineer, Quantity Surveyor, etc) and immersive environment for the end user to validate the design. The model data server and desktop clients were implemented using TechSoft's Hoops 3D Graphic Library, while the immersive environment utilized the OpenCOVER renderer and VICON tracking system. All these components were network-linked using the TechSoft's Hoops Net toolkit.

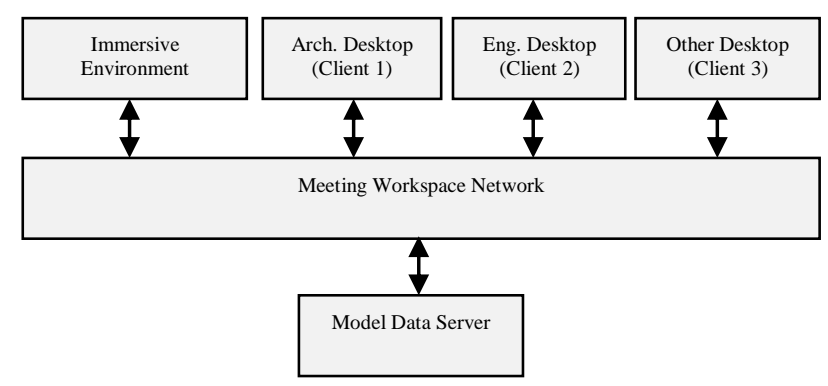

Figure 3 The core system components

\section{The Role of Immersive Virtual Reality}

This case study was a good example to illustrate the impact of the Immersive Virtual Reality on design meetings. Although the use of VR in this particular scenario was limited to test the design, its impact was huge to save the project cost and time. If the design proves to be wrong as discussed in the current practice, another meeting is required for another test with another physical mock-up.

The other added benefit of the digital mock-up is the visualisation aspect. The fact that the Immersive VR can simulate the experience of moving through and interacting with the virtual world as if it was real [15] enables the stakeholders to use the digital mock-up to perform any tests required during the meeting in order to validate any highlighted issues. Thus, VR produces a 
way for humans to visualise, manipulate and interact with computers and extremely complex data [16] Furthermore, irrespective whether VR is fully immersive, semi-immersive or non-immersive [17] [18], it provided the users with a sense of being there in the user's mind. This sense of 'being there' provided by realtime interaction, means that the system is able to detect a user's input and modify the virtual world instantly [19] .

Whether the digital mock-up is tested by a wheelchair user as seen in the futuristic scenario or by an Avatar as presented in figure 4, this case study illustrates the potential that Immersive Virtual Reality can have on visualisation of architectural design and the possibility of the digital model to replace the physical one which has been used for a very long time to enable both the architect as well as the client to visualise the building and its visual effect prior to construction [20] [21] .

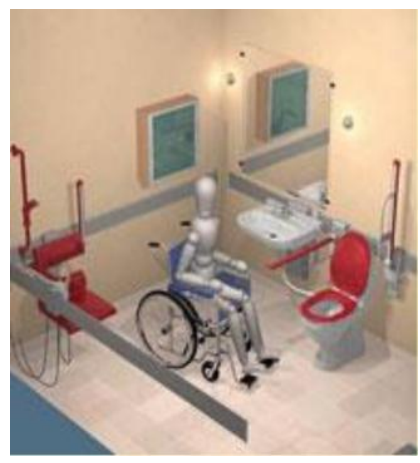

\section{Figure 4 An Avatar is used to test a 3D representation of a bathroom}

\section{Conclusions}

We described the impact that Immersive technologies can have on design projects through explaining the current practice (as is) and identifying some of the problems within the industry. We then envisaged a futuristic vision (to be) by using immersive technology in the same case study and examining the impact that technologies such as Immersive Virtual Reality can have on communication, decisions making and collaboration.

\section{Acknowledgements}

The results of this paper are partly funded by the European Commission under contract IST-5-034245 through the CoSpaces project. The authors would like to thank the industrial partners of the CoSpaces project and in particular we would like to thank COWI (Denmark) and Construct IT (UK) for their valuable information about current industrial practices and challenges.

\section{References}

[1] May Bassanino (1999) The Perception of Computer Generated Architectural Images, PhD Thesis, the University of Liverpool, UK.

[2] Shu Ling Lu, and Martin Sexton (2006) Innovation in Small Construction Knowledge-Intensive Professional Service Firms: A case Study of an Architectural Practice. "Construction Management and Economics", 24(12), 1269-1282.

[3] Patricia Montiel-Overall, (2005) Toward a theory of collaboration for teachers and librarians. "School Library Media Research", 8.

[4] May Bassanino, Bryan Lawson, John Worthington, Michael Phiri, Alastair Blyth and Chris Haddon (2001) 'Final Report: Learning from Experience- Applying systematic Feedback to improve the briefing process in construction', The University of Sheffield, Sheffield, UK.

[5] Alastair Blyth and John Worthington (2001) "Managing the Brief for Better Design". UK: Spon Press.

[6] Kurt Hanks and Larry Belliston (1992) Draw! A Visual Approach to Thinking, Learning and Communicating. Crisp Publications, Canada.

[7] Andy Brown and May Nahab (1996) Human interpretation of computer generated architectural images. In: Asanowicz and Jakimowicz (Ed). CAD and Creativity, Bialystok, TU Bialystok.

[8] Andy Brown, Mike Knight and May Nahab (1999) 'Computer Generated Architectural Images: a comparative study'. In: Brown A, Knight $\mathrm{M}$ and Berridge P (Ed.) Architectural Computing from Turning to 2000, 17th Conference of eCAADe, The University of Liverpool, Liverpool, UK.

[9] Paul Richens (1994) Does Knowledge Really Help? "Automation in Construction", 3(2-3), 219-227.

[10] Aart Bijl (1989) Computer Discipline and Design Practice. Edinburgh University Press. Edinburgh.

[11] Tsuyoshi Sasada (1995). Computer Graphics as a Communication Medium in the Design Process. In: Tan $M$ (ed.) Proceedings of the CAAD Futures'95. Singapore.

[12] CoSpaces url: http://www.cospaces.org/index.htm

[13] Gilles Gautier, Colin Piddington, May Bassanino, Terrence Fernando and Jens Skjærbæk (2008) 'Futuristic design review in the construction industry'. In: Zarli A and Scherer $\mathrm{R}$ (Ed.), eWork and eBusiness in Architecture, Engineering and Construction Conference, Sophia-Antipolis, France. Taylor and Francis Group, Vol. 1, pp. 625-633.

[14] May Bassanino, Gilles Gautier Kuo-Cheng Wu, Terrence Fernando, and Jens Skjærbæk (2009) 'An Innovation Approach to Improve Collaboration in a Futuristic Design Review'. In: McCaffer R (Ed.), Global Innovation in Construction Conference Proceedings, University of Loughborough, UK.

[15] Jialiang Yao (2010). A Collaborative Urban Planning Environment, PhD Thesis, The University of Salford, UK.

[16] Steve Aukstakalnis and David Blatner (1992). Silicon Mirage: The Art and Science of Virtual Reality, Peachpit Press.

[17] Neil Dodgson (2005). Autostereoscopic 3D Displays. IEEE Computer 38(8):31-36. 
[18] Intuition Virtual Reality Final Report (2007), IST-NMP1-507248-2, Future Workspaces Research Centre, The University of Salford.

[19] Grigore Burdea and Philippe Coiffet (2003). Virtual Reality Technology, Wiley-IEEE Press.

[20] Tom Porter (1997). The Arxchitect's Eye. E\&FN Spon. UK.

[21] Martin Fischer (2008) Reshaping the life cycle process with virtual design and construction methods. In: Brandon P. and Kocaturk T. (eds.) "Virtual Futures for Design, Construction \& Procurement". Oxford: Blackwell Publishing Ltd. 